Vercellone, A. L. ¿Puede la administración pública ser un agente autónomo? Una crítica a la teoría del órgano desde la filosofía moral. Derecho y Ciencias Sociales. Mayo-Octubre 2019 № 21. Pgs 166-185. ISNN 1852-2971. Instituto de Cultura Jurídica y Maestría en Sociología Jurídica. FCJ y S. UNLP

\title{
¿Puede la administración pública ser un agente autónomo? Una crítica a la teoría del órgano desde la filosofía moral
}

Can public administration be an autonomous agent? A moral philosophical critique on organicist theory

\section{Adriana L. Vercellone}

\section{Resumen}

Este artículo considera la justificación de atribuir responsabilidad jurídica a los entes colectivos, especialmente, a la administración pública. En concreto, se evalúa si es dable entender a la administración pública como un agente autónomo, diferente de las personas que la conforman. Para ello, se hace un recorrido por los argumentos de las dos principales concepciones teóricas elaboradas en torno al tema: la teoría del órgano, que es la más aceptada en el ámbito de los juristas; y el estructuralismo, que es la más novedosa teoría filosófica sobre responsabilidad grupal. Las conclusiones muestran que los presupuestos teóricos que defiende la teoría del órgano no son admisibles para el estudio de la atribución de responsabilidad en el ámbito público.

Palabras clave: responsabilidad colectiva, teoría del órgano, administración pública, estructuralismo.

\begin{abstract}
This article analyses collective legal responsibility, particularly in public administration. The main objective is to consider whether public administration it is or it is not an autonomous agent, different from its members. In order to accomplish this, the article analyses the two main theoretical conceptions developed in the subject: the organicist theory, which is the most accepted in law; and structuralism, which is the most accepted philosophical theory about collective responsibility. We conclude that some theoretical assumptions that organicists defend, cannot be admited in legal responsibility systems for public sphere.
\end{abstract}

Key words: collective responsibility, organicist theory, public administration, structuralism.

\footnotetext{
-Doctora en Derecho y Ciencias Sociales (Universidad Nacional de Córdoba), Magister en Derecho y Argumentación (UNC), Abogada (UNC). Profesora en "Ética” y "Derecho Político" en Facultad de Derecho (UNC). Investigadora en Programa de ética y teoría política (Centro de Investigaciones Jurídicas y Sociales, UNC). Correo electrónico: adrivercellone@gmail.com. Doctora en Derecho y Ciencias Sociales (Universidad Nacional de Córdoba), Magister en Derecho y Argumentación (UNC), Abogada (UNC). Profesora en "Ética" y "Derecho Político" en Facultad de Derecho (UNC). Investigadora en Programa de ética y teoría política (Centro de Investigaciones Jurídicas y Sociales, UNC). Correo electrónico: adrivercellone@gmail.com
}

Recibido 22/10/2018. Publicable con correcciones 2/9/2019 
Vercellone, A. L. ¿Puede la administración pública ser un agente autónomo? Una crítica a la teoría del órgano desde la filosofía moral. Derecho y Ciencias Sociales. Mayo-Octubre 2019 № 21. Pgs 166-185. ISNN 1852-2971. Instituto de Cultura Jurídica y Maestría en Sociología Jurídica. FCJ y S. UNLP

\section{¿Puede la administración pública ser un agente autónomo? Una crítica a la teoría del órgano desde la filosofía moral}

Adriana L. Vercellone ${ }^{1}$

\section{Introducción}

Nuestra legislación reconoce a los entes colectivos la potestad de contraer derechos y obligaciones confiriéndoles el trato de persona jurídica. ${ }^{2}(C C C N$ : art141). En base a esto, los entes constituyen un agente independiente de las personas que lo componen, con la potestad de ser responsables por las actividades que despliegan. En ese sentido, nuestro sistema legal se ha acoplado a una tendencia ya vigente en otros ordenamientos jurídicos que asimilan el estatus de dichos entes al de las personas físicas.

El argumento más recurrido para explicarlo ha sido la teoría del órgano. Ésta entiende a la persona jurídica como un organismo de cual sus integrantes son órganos; y así interpreta a las acciones de sus directores, administradores o dependientes como si procedieran de la misma voluntad del ente. Es una teoría identificada dentro de las llamadas estructuralistas, pues sostienen que siendo la persona física un órgano de expresión del ente, la acción no es imputada individualmente a la persona física que la llevó adelante sino a la estructura organizativa como tal.

Esta línea argumentativa implica, entre otras cosas, que cuando nuestro CCCN define a las personas jurídicas como sujeto de derecho, entiende que ésta constituye un centro volitivo autónomo. O que, al atribuir al ente colectivo la responsabilidad por los hechos de sus dependientes, éstos últimos no actúan en nombre propio ni siguiendo propios objetivos, sino como una mera función del ente.

Sin embargo, para que un grupo sea calificado como un agente autónomo debe satisfacer ciertos requisitos específicos. Una de las teorías filosóficas contemporáneas que mejor explica estos requisitos es el estructuralismo de Philip Pettit. De acuerdo con ella, un grupo sólo puede constituirse como agente autónomo cuando su estructura interna satisface ciertos estándares democráticos y deliberativos. Por el contrario, cuando la organización no adopta dichos criterios, ésta no es más que la mera suma de los individuos que lo componen sin objetivos, juicios o acciones propias.

\footnotetext{
${ }^{1}$ Agradezco a Cristián Fatauros por sus valiosas observaciones a una versión preliminar de este artículo, y a Hugo Seleme por participar en la discusión de algunas ideas centrales.
} 
Vercellone, A. L. ¿Puede la administración pública ser un agente autónomo? Una crítica a la teoría del órgano desde la filosofía moral. Derecho y Ciencias Sociales. Mayo-Octubre 2019 № 21. Pgs 166-185. ISNN 1852-2971. Instituto de Cultura Jurídica y Maestría en Sociología Jurídica. FCJ y S. UNLP

Un análisis detallado sobre la teoría de Pettit permite extraer valiosas conclusiones en relación con la teoría del órgano y el ordenamiento jurídico local sobre responsabilidad. La primera de ellas es que no cualquier grupo humano satisface los parámetros para constituirse como agente autónomo. Esto es especialmente relevante cuando consideramos la actividad que despliega la administración pública, por el tipo de vínculos intersubjetivos que mantienen las personas en su interior -ciudadanía y quienes ejercen cargos públicos -, y por los principios burocráticos que suelen organizarla. Al analizar la plausibilidad de este argumento en el contexto estatal se hace evidente una segunda conclusión: que la administración pública difícilmente satisface los requisitos que el estructuralismo exige.

Si se aceptan ambas conclusiones, entonces es necesario considerar la verdadera plausibilidad de algunos principios legales que tratan a las instituciones públicas como personas jurídicas. $\mathrm{O}$ en su caso, reflexionar sobre la teoría del órgano como la tesis que por defecto se ha usado para justificar los supuestos de responsabilidad colectiva.

Este artículo intenta explicar y justificar estas conclusiones. Para ello, primero se reconstruyen los principios generales sobre responsabilidad colectiva y la teoría del órgano que se encuentra detrás de parte importante de nuestro entramado jurídico. Luego, se analiza el argumento estructuralista de Pettit intentando mostrar sus ventajas y problemas. Por último, se evalúa la posibilidad de que ambos argumentos teóricos justifiquen las actividades de la administración pública. El objetivo último del artículo es resaltar las implicancias jurídicas y prácticas que tiene una respuesta negativa sobre el tema.

\section{Las personas jurídicas y la teoría del órgano en nuestro sistema legal}

La posibilidad de que los entes colectivos constituyan un centro de razones autónomo -diferente a sus miembros- ha sido profusamente discutida en el ámbito jurídico. En nuestro medio, dicho debate ha estado presente tanto en el derecho civil, como en el derecho comercial, el régimen penal y el derecho administrativo. Aunque la discusión persiste a nivel teórico, la legislación ha avanzado en conceder a los grupos humanos la potestad de contraer derechos y obligaciones con similar alcance que las personas físicas, aunque por las limitaciones propias de su naturaleza colectiva necesite de personas que actúen en su nombre. Esto se ha plasmado, principalmente, en los artículos 145 y 146 del CCCN, y parte importante de la legislación civil y comercial complementaria. ${ }^{3}$

\footnotetext{
${ }^{3}$ Ver principalmente, Ley de Responsabilidad el Estado $N^{\circ} 26.944$ (LRE) y Ley de Sociedades Comerciales $\mathrm{N}^{\circ}$ 19.550 (LSC).
} 
Vercellone, A. L. ¿Puede la administración pública ser un agente autónomo? Una crítica a la teoría del órgano desde la filosofía moral. Derecho y Ciencias Sociales. Mayo-Octubre 2019 № 21. Pgs 166-185. ISNN 1852-2971. Instituto de Cultura Jurídica y Maestría en Sociología Jurídica. FCJ y S. UNLP

Por su parte, en el ámbito penal ha predominado la posición contraria. Bajo el adagio nullen crimen sine conducta se asume que sólo las personas físicas pueden actuar u omitir actuar, y así las personas jurídicas no pueden cometer delitos ni caer bajo la órbita de aplicación de la ley penal(Cesano \& Balcarce, 2003; Robiglio, 2007). ${ }^{4}$ Dicha tesis es sostenida, inclusive, por quienes admiten que los entes sean sancionados, pues uno de sus argumentos fuertes es la peligrosidad de las personas jurídicas. Así, sólo consideran la aplicación de sanciones reparadoras al grupo con el objeto de competir contra el crimen organizado, la corrupción o meramente para proteger al ciudadano de quien se encuentra en una posición favorecida por el contexto económico o social, como por ejemplo una gran empresa; pero no atribuyéndole la capacidad de ser un centro de razones autónomo. ${ }^{5}$

La teoría del órgano es la más aceptada entre los juristas como antecedente de justificación de los supuestos de responsabilidad grupal mencionados. ${ }^{6} \mathrm{Su}$ presupuesto fundamental es que la persona jurídica es un organismo del que sus integrantes son órganos, y así interpreta a las acciones de sus directores, administradores o dependientes como si procedieran de la misma voluntad del ente colectivo.

En concreto, cuando una organización causa un daño surgen diferentes preguntas sobre la imputación: a) quién debe responder, b) bajo qué condiciones la acción individual puede ser atribuida al grupo, y c) si la responsabilidad grupal e individual son excluyentes entre sí. Como respuesta, la teoría del órgano explica las condiciones que deben darse para que un evento pueda serle atribuido al ente colectivo. En ese sentido, es básicamente una "teoría sobre la imputación" (Valdivia Olivares, 2006) que considera que:

A. La persona jurídica sólo puede ejercer sus capacidades por medio de sus representantes legales;

B. La acción estatal es imputada al ente, y no individualmente al agente público que la llevó adelante;

C. Aquello a lo que puede quedar obligado el Estado queda delimitado por la autorización o el poder conferido al agente individual actuante.

\footnotetext{
${ }^{4}$ Por ejemplo, ver voto en disidencia del Juez Zaffaroni en "Dirección General de Aduanas c. Fly Machine SRL" CJSN, 2006. En un escrito que ha marcado tendencia, Zaffaroni asume dicho principio, no obstante reconocer la posibilidad de que las personas jurídicas sean sometidas a sanciones administrativas o reparadoras.

5 Inclusive, las excepciones de responsabilidad colectiva que se reconocen -por ejemplo, en la ley de estupefacientes, el régimen penal cambiario y la ley aduanera- no se han previsto para entes públicos, sino que son propias del derecho penal económico, y tampoco están exentas de discusión (Robiglio, 2007, p. 9).

${ }^{6}$ Se cita esta tesis, ya que es la que cuenta con acuerdo mayoritario en nuestro medio. Aunque vale mencionar que la doctrina también distingue otras menos recurrida: teoría de la ficción, teoría del mandato, teoría de la representación y teoría de la institución (Echevesti, 2003).
} 
Vercellone, A. L. ¿Puede la administración pública ser un agente autónomo? Una crítica a la teoría del órgano desde la filosofía moral. Derecho y Ciencias Sociales. Mayo-Octubre 2019 № 21. Pgs 166-185. ISNN 1852-2971. Instituto de Cultura Jurídica y Maestría en Sociología Jurídica. FCJ y S. UNLP

Las consideraciones sobre esta teoría son especialmente relevantes al reflexionar sobre el ámbito público. Siendo el Estado Nacional una "persona jurídica de carácter público” se ha reconocido como principio general que el Estado responde de forma objetiva y directa por los daños que causa la administración pública en su actividad lícita e ilícita. Aunque se admiten excepciones en los casos de culpa o dolo del agente público, no se discute que todas las personas integradas a la estructura del Estado pueden obligarlo, y que dicha obligación queda delimitada por la autorización o el poder que se ha conferido al representante (Ley de Responsabilidad del Estado: arts 2 y 6).Asimismo, se acepta que la responsabilidad directa es un claro indicio de reconocimiento al Estado de la posibilidad de actuar y decidir autónomamente (Cassagne, 2005; Palazzo, 1995; Pizarro, 2016).

En otras palabras, la teoría del órgano asume que la administración pública es un ente constituido por el conjunto de personas que conforman la comunidad política -ciudadanía y quienes ejercen cargos públicos-. Sus fines y objetivos son propios e independientes, y cuenta con plena capacidad para actuar en coincidencia con ellos, aunque necesite de personas físicas para ejecutarlos. Siempre que sus miembros actúen dentro de los márgenes y fines previstos legalmente, la administración responde por la actividad pública que desarrolla. Las acciones regulares y normales de cualquier agente público son imputadas a la administración pública como si de ella procedieran, y el funcionario sólo es responsable a título individual cuando se ha excedido o ha desobedecido mandatos recibidos. ${ }^{7}$

En definitiva, y aunque existen diversas versiones de la teoría del órgano ${ }^{8}$, esta parece apoyarse en un presupuesto común: que los entes colectivos pueden constituir un centro volitivo autónomo, diferente de sus miembros. Aunque sus defensores no profundizan en la noción de agencia moral y autonomía grupal, es un tema que la filosofía moral sí ha estudiado profusamente; principalmente el estructuralismo.

\section{La responsabilidad de los grupos en la filosofía}

\footnotetext{
${ }^{7}$ Existen múltiples distinciones entre el tipo de actividades que pueden o no generar responsabilidad al Estado. Por mencionar algunos, se sostiene que el Estado sólo responde por su actividad irregular o ilícita, aunque también se ha reconocido responsabilidad por lícitos cuando el ciudadano perjudicado no tiene el deber de soportar el daño; asimismo, que ante culpa, dolo o negligencia del agente público, éste responde por los daños con su propio patrimonio (Andrada, 2010; Cassagne, 2005; Echevesti, 2003; Hevia, 2014; Ivanega, 2005; Palazzo, 1995; Pizarro, 2016; Trigo Represas, 2009). Pero pese a estas distinciones, el principio general sobre responsabilidad no se modifica; y la teoría del órgano que lo justifica tampoco.

${ }^{8}$ Se reconocen dos versiones de la teoría: subjetiva y objetiva. Es decir que, dentro de los mismos organicistas, algunos juristas defienden un estructuralismo irrestricto, mientras otros un tipo de estructuralismo compatible con la responsabilidad subjetiva del agente individual (Echevesti, 2003).
} 
Vercellone, A. L. ¿Puede la administración pública ser un agente autónomo? Una crítica a la teoría del órgano desde la filosofía moral. Derecho y Ciencias Sociales. Mayo-Octubre 2019 № 21. Pgs 166-185. ISNN 1852-2971. Instituto de Cultura Jurídica y Maestría en Sociología Jurídica. FCJ y S. UNLP

Entre los filósofos que defienden la responsabilidad colectiva es usual distinguir las siguientes posiciones (Feinberg, 1970).

La primera atribuye responsabilidad al grupo por la falta de uno o algunos de sus miembros. Esta tesis sostiene que responden todos los miembros del grupo porque comparten vínculos de solidaridad o una comunidad de interés, tal lo que sucede con los ejércitos militares, un grupo religioso o inclusive un grupo familiar (Risser, 2006).

La segunda atribuye responsabilidad al grupo por una falta contributiva o no contributiva. Aunque la falta ha sido sólo de uno o varios de los miembros del grupo, la culpa es común atento algún defecto de carácter que todos comparten. Por ejemplo, un grupo cuyos miembros acostumbran alcoholizarse pero sólo uno o varios causan un daño (Risser, 2006).

Una tercera posición considera que el grupo es responsable por la falta contributiva de todos sus miembros, entendiendo que todos han contribuido con su acción en la falta cometida. Por ejemplo, los colectivos espontáneos (como un conjunto de personas que ocasionalmente comparten espacio en el transporte público o los espectadores en la cancha de un partido de fútbol) y las agrupaciones formales o con cierta estructura interna formal, como una empresa privada o pública. El rasgo en común es que los miembros del grupo pueden variar en el tiempo y no comparten vínculos de solidaridad, más allá de la actividad común que regularmente despliegan (Risser, 2006).

Algunas de estas teorías han sido criticadas por su extremo reduccionismo, pues reducen toda actividad grupal a la mera suma de sus individuos; mientras que otras han sido señaladas como emergentes, al atribuira los entes colectivos un estado metafísico diferente al de sus miembros (List \& Pettit, 2011, pp. 73-74). Es decir que mientras unas pecan por reducir la estructura grupal solo a los individuos que deliberan y actúan motivados por el propio interés, las otras atribuyen a los entes la misma entidad y autonomía que a las personas físicas, con los riesgos prácticos que ello implica(Thompson, 1999, 2004).

En contraposición, se defiende una cuarta posición según la cual los entes colectivos pueden ser responsables de formaindependiente respecto de cada uno de sus miembros por la falta colectiva pero no distributiva (Risser, 2006). Es decir que la falta se atribuye a la estructura del grupo, como un agente autónomo, dada su constitución y estructuras internas. En esta línea, el estructuralismo de Pettit sugiere que los grupos organizados pueden ser vistos como algo más que la conjunción de sus miembros y constituir una entidad moral en sí misma -aunque no metafísica-, lo cual permite atribuirle responsabilidad por sus hechos pasados y predecir su futura actividad. 
Vercellone, A. L. ¿Puede la administración pública ser un agente autónomo? Una crítica a la teoría del órgano desde la filosofía moral. Derecho y Ciencias Sociales. Mayo-Octubre 2019 № 21. Pgs 166-185. ISNN 1852-2971. Instituto de Cultura Jurídica y Maestría en Sociología Jurídica. FCJ y S. UNLP

\section{La teoría estructuralista}

El estructuralismo de Philip Pettit constituye una de las explicaciones más sofisticadas de los principios y consecuencias de la responsabilidad colectiva. Su argumento se desglosa en dos preguntas centrales. ¿Qué caracteriza a los agentes morales? Dadas las múltiples formas que adquieren las acciones grupales, la segunda interroga ¿bajo qué condiciones la performance de los grupos es equivalente al comportamiento de un agente moral?

\section{a. Tesis sobre la agencia}

Para Pettit los agentes morales comparten tres rasgos:

a) estados representacionales, que suponen la capacidad de representarse cómo está configurado el mundo,

b) estados motivacionales, que explican como debiera ser el contexto o el ambiente, de manera tal que pueden motivar la acción, y

c) la capacidad de procesar y actuar como consecuencia de (a) y (b); es decir, la capacidad de procesar ambos estados e inducir al agente a intervenir en el mundo cuando éste no se ajusta a las motivaciones que tiene en virtud de sus representaciones (List \& Pettit, 2011, pp. 19-42).

Estos tres rasgos describen a un típico agente intencional, reconociendo que estos "[...] interactúan con su ambiente bajo el control de regularidades intencionales" (Pettit, 1996, p. 11).Para reforzar la idea, Pettit agrega que lo verdaderamente distintivo en el agente moral es la capacidad de tener creencias y deseos acerca de las propiedades del mundo, de los estados representacionales y motivacionales, y de las mismas proposiciones. Básicamente, esta capacidad es el razonamiento y permite a la persona "reforzar su racionalidad" (List \& Pettit, 2011, p. 30), por ejemplo, cuestionándose sobre la verdad de sus proposiciones o formulando preguntas abstractas sobre sus motivaciones. Esta capacidad de formular deseos y creencias de segundo orden es fundamental para distinguir la mera racionalidad de las cualidades que tiene la persona humana. Por ejemplo, un animal que no responde a un estímulo de su entorno cuando éste no se ajusta a sus estados representacionales o motivacionales adolece de uno de los rasgos fundamentales de la agencia. En cambio, una persona que comete dicha falta puede más adelante evaluar su comportamiento como equivocado, corregirlo o cuestionarlo, es decir, elaborar proposiciones o preguntas de segundo orden sobre su propio comportamiento. Por ello, se insistirá en que es un agente moral (List \& Pettit, 2011, p. 31). 
Vercellone, A. L. ¿Puede la administración pública ser un agente autónomo? Una crítica a la teoría del órgano desde la filosofía moral. Derecho y Ciencias Sociales. Mayo-Octubre 2019 № 21. Pgs 166-185. ISNN 1852-2971. Instituto de Cultura Jurídica y Maestría en Sociología Jurídica. FCJ y S. UNLP

\section{b. Acciones grupales y agencia grupal}

Para que un grupo humano manifieste los rasgos de agencia moral, sus actividades y decisiones deben ser verdaderamente desplegadas de forma conjunta. Que no se trate de una actividad conjunta realizada a ciegas es fundamental, ya que Pettit observa cuan común es que los grupos causen resultados en el mundo de forma espontánea: muchas veces desplegando actividades individuales que de forma aleatoria están interconectadas, otras con el designio de realizar la actividad de forma conjunta por parte de sólo algunos de sus miembros. Por esto, cree que es importante distinguir casos en los que dichos resultados pueden ser atribuidos a un agente grupal de otros en los que es atribuible simplemente a la suma de voluntades individuales. En ese sentido, afirma que la importancia está en hablar apropiadamente de la “[...] acción conjunta, y no de que han producido simplemente un efecto conjunto"(Pettit \& Schweikard, 2006, p. 3). Esto implica considerar a la acción no como una mera función grupal intencional para evaluarla como algo diferente que constituye a un agente.

Esto es posible cuando el grupo presenta los siguientes: (List \& Pettit, 2011, pp. 33-39)

i. Objetivo compartido: que existe cuando las personas que conforman el grupo se enfocan en la performance conjunta de la acción, con la noción de que dicha acción constituye un fin común (Pettit \& Schweikard, 2006, p. 6).

ii. Contribución individual: que se da cuando cada individuo contribuye en la actividad grupal, sea autorizando la acción o siendo un actor de ella.

iii. Interdependencia: que exige plena consciencia de que los otros miembros del grupo realizan una contribución individual a la acción conjunta, y de que cada uno lleva a cabo la acción para el cumplimiento del fin común.

iv. Noción de la satisfacción de los primeros tres elementos: que exige conocimiento y convicción de que todos los miembros del grupo satisfacen las tres anteriores.

Aunque la mayoría de los grupos humanos formalmente constituidos parecen presentar los cuatro elementos, en realidad no siempre es así. En especial, porque los agentes individuales suelen comportarse de forma autointeresada o irracional dentro de los grupos. Por ejemplo, cuando una o varias personas vician las decisiones colectivas para el logro de fines personales, en cuyo caso no se satisface la exigencia de "objetivo compartido". O cuando, aun siendo las personas racionales y comprometidas colectivamente, la estructura organizacional no despliega juicios o actividades consistentes. Es decir que, las personas podrían no pensar en términos individuales y tener la firme voluntad de constituir un centro intencional plural, y aun así obstaculizar que el grupo pueda sostener juicios racionales. 
Vercellone, A. L. ¿Puede la administración pública ser un agente autónomo? Una crítica a la teoría del órgano desde la filosofía moral. Derecho y Ciencias Sociales. Mayo-Octubre 2019 № 21. Pgs 166-185. ISNN 1852-2971. Instituto de Cultura Jurídica y Maestría en Sociología Jurídica. FCJ y S. UNLP

En otras palabras, la agencia moral grupal puede ser obstruida por vicios individuales o por defectos en la estructura colectiva. En definitiva, lo que determina la posibilidad de "verdadera" agencia moral colectiva es el modo en que se vinculan las voluntades individuales con la voluntad grupal. En este punto el estructuralismo de Pettit es una teoría sumamente sofisticada, porque para responder a estas cuestiones formula un argumento que se entiende superador de las tesis rivales: aquel que define a la relación entre el grupo y los individuos como una relación de superveniencia(List \& Pettit, 2005, 2011).

\section{c. La tesis de la superveniencia y la estructura de los grupos que tienen agencia}

Como se indicó, para que sea posible la agencia moral grupal debe existir un tipo de relación apropiada entre voluntades individuales y voluntad grupal. Para explicar este vínculo Pettit sostiene que "una serie de hechos, digamos B, superviene en otros, $A$, si y sólo si, necesariamente, disponer los hechos de A también dispone los de B”(List \& Pettit, 2011, p. 65).

Como esta relación puede adoptar diversas formas, Pettit se propone mostrar las tesis implausibles.

La primera tesis que describe es la "superveniencia mayoritaria", que define el juicio grupal respecto de una proposición en base a lo que la mayoría de los miembros del grupo sostiene sobre dicha proposición (List \& Pettit, 2011, p. 10).

Esta relación mayoritaria puede devenir en juicios grupales inconsistentes, como muestra el dilema discursivo; es decir, la paradoja que se suscita cuando un colectivo debe decidir sobre una cuestión cuyo resultado mayoritario se superpone o es contradictorio con lo que la mayoría de los agentes individuales sostiene en cada una de las premisas que la componen (Pettit, 2001a). En este caso, los juicios del grupo son inconsistentes porque alguna de las proposiciones sometidas a decisión es afirmada y negada de modo simultáneo (List \& Pettit, 2011, p. 8).

Un ejemplo que lo explica es la decisión del Congreso sobre la posibilidad de invertir en medidas de seguridad para un determinado trabajo. Pettit imagina un grupo (conformado por agentes $\mathrm{A}, \mathrm{B}$ y C) que debe decidir sobre tres premisas diversas: si existe un peligro en la tarea que tienen encomendado realizan, la efectividad de las medidas a adoptar para su efectiva realización, y la posibilidad de que las personas costeen dichas medidas. El requisito de consistencia es que, sólo cuando la respuesta a las tres cuestiones es positiva, entonces votarán por invertir en dicha medida (Pettit, 2003, p. 143).

Una posible matriz de votación es la siguiente: 
Vercellone, A. L. ¿Puede la administración pública ser un agente autónomo? Una crítica a la teoría del órgano desde la filosofía moral. Derecho y Ciencias Sociales. Mayo-Octubre 2019 № 21. Pgs 166-185. ISNN 1852-2971. Instituto de Cultura Jurídica y Maestría en Sociología Jurídica. FCJ y S. UNLP

$\begin{array}{lllll} & \text { Peligro } & \text { Efectividad } & \text { Costo } & \text { Invertir } \\ \text { A. } & \mathrm{Si} & \mathrm{No} & \mathrm{Si} & \text { No } \\ \text { B. } & \mathrm{No} & \mathrm{Si} & \mathrm{Si} & \text { No } \\ \text { C. } & \mathrm{Si} & \mathrm{Si} & \mathrm{No} & \text { No } \\ \text { Mayoría. } & \mathrm{Si} & \mathrm{Si} & \mathrm{Si} & \text { No }\end{array}$

El dilema que revela el caso es el siguiente: lo que la mayoría de los individuos considera para las premisas individualmente consideradas (que hay peligro, que las medidas serían efectivas y que las personas pueden soportar el costo) es inconsistente con lo que la mayoría cree respecto de la inversión (que no debe realizarse). Aun tratándose de agentes que sostienen individualmente juicios racionales completos y consistentes (ya que nadie afirma la conclusión si no ha afirmado sus tres premisas anteriores), lo que representan a nivel grupal no lo es. Y no lo es por una falencia en el mecanismo de adopción de decisiones: meramente mayoritario.

Pettit describe además dos tesis alternativas, una en la que la función no es mayoritaria (“superveniencia uniforme sobre las proposiciones”), y otra en la que la función varía según la premisa sometida a decisión ("superveniencia sobre las proposiciones"). Pero el problema de inconsistencia aparece siempre que el juicio grupal es el resultado o función de los juicios individuales. Aún en los casos en los que esta función no es mayoritaria -por ejemplo, cuando se exige unanimidad-, o cuando se alterna el requisito para cada premisa -por ejemplo, se exige mayoría para decidir sobre las premisas, y unanimidad para la conclusión- (List \& Pettit, 2011, pp. 11-14). Por esto, la conformación de la voluntad grupal debe seguir alguna función de superveniencia más compleja.

Así, piensa en una relación de superveniencia que:

- Disponga las actitudes del grupo de forma independiente a la conformación de sus miembros, pues de lo contrario se trataría de una relación de determinación -es decir, un caso en el que la voluntad individual determina la grupal-.

- Se caracterice por actitudes grupales racionales, consistentes y completas, para que puedan considerarse el producto de un ejercicio de racionalidad grupal.

En virtud de ambas consideraciones enuncia la máxima de "racionalidad grupal robusta", que sostiene que "[...] la relación de superveniencia determina actitudes grupales completas y consistentes en las proposiciones relevantes para cualquier perfil posible de actitudes completas y consistentes de los miembros sobre esas proposiciones” (List \& Pettit, 2011, p. 67). 
Vercellone, A. L. ¿Puede la administración pública ser un agente autónomo? Una crítica a la teoría del órgano desde la filosofía moral. Derecho y Ciencias Sociales. Mayo-Octubre 2019 № 21. Pgs 166-185. ISNN 1852-2971. Instituto de Cultura Jurídica y Maestría en Sociología Jurídica. FCJ y S. UNLP

Para que esta máxima se realice en un grupo la actitud grupal no puede ser simplemente lo que la mayoría de los miembros cree respecto de una proposición, ni lo que cada individuo cree sobre cada proposición en agenda, sino que debe ser "holística". Así,"el grupo de actitudes a través de las proposiciones es determinado por el conjunto de actitudes individuales a través de las proposiciones "(List \& Pettit, 2011, pp. 68-70).

Es más fácil entender el argumento en la práctica de los grupos. En cuyo caso, la máxima implica la adopción de regulaciones para la toma de decisión grupal con el objetivo de evitar los peligros que antes enunció: evitar que la voluntad de uno o varios individuos determine de forma aislada el juicio grupal, y asegurar consistencia grupal.

\section{d. Diseños y regulaciones que permiten agencia moral grupal}

Además del problema de imposibilidad de asociación (dilema discursivo) que antes describió, Pettit encuentra un nuevo "enemigo": las estructuras formales de la organización (Pettit, 2001a, 2003). Ya que cuando hay división de tareas, no todos los agentes comparten el objetivo grupal ni tienen noción de la persecución de dicho objetivo como algo en común. Por ello, es difícil que las creencias puedan ser sostenidas y conocidas por todos los individuos del grupo. En definitiva, no cualquier grupo tiene autonomía de acción y existen estructuras que fomentan lo contrario a ella.

Para garantizar juicios racionales y consistentes, Pettit imagina dos "rutas": una "organizacional" que promueve conductas cooperativas y veraces a través de mecanismos organizacionales, y una "conductual" que promueve la modificación de preferencias individuales a través de procesos de educación o persuasión (List \& Pettit, 2011, pp. 124-128). Ejemplos de ambas son los estatutos internos que exigen deliberación grupal sobre los valores en juego, y las instancias de formación o sistemas creativos de premios que una institución puede instalar.

Frente al problema de consistencia y completitud de los juicios grupales, destaca las siguientes regulaciones estructurales. La primera es la democratización. Según ella, siendo las opiniones de los miembros del grupo “independientes entre sí” e "inclinadas hacia la verdad" (es decir, asumiendo que las proposiciones que las personas adoptan son de competencia ideal), la probabilidad de que el resultado final se acerque a la verdad sucede: a) cuando se aplica la regla de la mayoría, y b) a medida que el grupo aumenta su número (List \& Pettit, 2011, pp. 82-86). Pero cuando las condiciones no son ideales, la opción es conceder voto diferenciado a algunos agentes individuales -los mejor calificados- (o estrategia de descomposición). Es decir, que la estructura prevea si todos los individuos contribuyen del mismo modo o tienen diferente 
Vercellone, A. L. ¿Puede la administración pública ser un agente autónomo? Una crítica a la teoría del órgano desde la filosofía moral. Derecho y Ciencias Sociales. Mayo-Octubre 2019 № 21. Pgs 166-185. ISNN 1852-2971. Instituto de Cultura Jurídica y Maestría en Sociología Jurídica. FCJ y S. UNLP

influencia en las decisiones (List \& Pettit, 2011, pp. 92-95). Por ejemplo, un estatuto interno podría conceder a algunos un voto de mayor peso.

Asimismo, el grupo podría establecer regulaciones al procedimiento de decisión. Por ejemplo, adoptar algún "límite lógico" indicando que, aunque vota mayoritariamente, acepta la conclusión sólo cuando se han afirmado las premisas anteriores. O establecer una secuencia determinada para la decisión sobre las premisas, por ejemplo, que sólo decidirá un tema $\mathrm{X}$ si antes han decidido sobre los temas A y B. O establecer doble ronda de votación, es decir, votar una vez por cada premisa, y luego de controlar los resultados, proceder a votar nuevamente para decidir sobre la conclusión. Así, se asegura que la conclusión sólo es afirmada si las premisas también han sido aseveradas.

Una última posibilidad es subdividir múltiples subgrupos de individuos (descentralización), de manera que cada uno se especialice en la toma de decisión mayoritaria sobre una premisa (unos en $\mathrm{P}$, otros en $\mathrm{Q}$, etc.). De esta forma la conclusión deriva de los juicios grupales en cada una de las premisas, aceptando también el límite lógico antes mencionado(List \& Pettit, 2011, pp. 9597).

Estas regulaciones impiden inconsistencias y garantizan juicios completos y deductivamente cerrados. Asimismo, permiten reconocer un vínculo entre las voluntades individuales y la colectiva que, independientemente del modo en que se conforma a nivel individual el grupo, posibilita juicios grupales consistentes y racionales:

- $\quad$ Las voluntades individuales supervienen en el grupo porque, si cambian las propiedades del grupo, es porque se han modificado las propiedades de los individuos que están en la base.

- $\quad$ Sin embargo, la regla sigue siendo que el juicio grupal no es generado ni determinado por ningún juicio individual.

En ese sentido, se suele decir que lo distintivo de este tipo de relación es que no es simétrica (Espí, 2011). De manera que dos grupos podrían tener el mismo fin o realizar una acción semejante, sin que los individuos que los componen tengan similares voluntades. Asimismo, los elementos del grupo -voluntades individuales- son suficientes para caracterizar la voluntad grupal, aunque no necesarios, ya que pueden existir otros casos de agencia grupal con conformación individual diversa. ${ }^{9}$

\footnotetext{
${ }^{9}$ Cabe mencionar el defecto que tienen las regulaciones propuestas: que el juicio grupal no es una función sólo de los juicios individuales, sino que puede ser atribuida a otras premisas y a los límites lógicos impuestos (List \& Pettit, 2005, pp. 15-18). En definitiva, ningún juicio individual es necesario para acceder a la conclusión y, en los hechos, las decisiones colectivas podrían ser impedidas o censuradas por las mismas regulaciones. En ese sentido, Pettit señala que un problema ulterior del dilema discursivo es la opción entre resignar democracia (regulando o limitando
} 
Vercellone, A. L. ¿Puede la administración pública ser un agente autónomo? Una crítica a la teoría del órgano desde la filosofía moral. Derecho y Ciencias Sociales. Mayo-Octubre 2019 № 21. Pgs 166-185. ISNN 1852-2971. Instituto de Cultura Jurídica y Maestría en Sociología Jurídica. FCJ y S. UNLP

\section{e. La responsabilidad de los grupos}

Pettit no duda que aquellos grupos que satisfacen los requisitos para constituirse como agentes morales, puedan ser responsables por las actividades que despliegan y sus decisiones. Y esto es porque, la capacidad de razonar augura: a) la posibilidad de enfrentar decisiones morales de relevancia, en definitiva, "él o ella es un agente autónomo y enfrenta una decisión de valor relevante que involucra la posibilidad de hacer algo bueno o malo, correcto o incorrecto"(Pettit, 2007, p. 175); b) pleno conocimiento en la culpa o, mejor dicho, que el agente se encuentra en posición de reconocer los valores que están en juego en una decisión determinada; y c) consentimiento de la acción, en el sentido de que la elección realizada realmente dependía del agente en cuestión, pues "La persona tiene el control necesario para ser capaz de elegir entre opciones sobre la base de un juicio acerca de su valor"(Pettit, 2007, p. 175).

La responsabilidad grupal, no implica desconocer que el ente actúa por medio de personas físicas. Para explicar que individuos y grupos puedan tener simultáneamente control sensible de las elecciones grupales, aunque en diferente nivel, defiende la existencia de una constitución o estatuto que organice al grupo y ordene las conductas individuales y las prácticas de acuerdo a tareas que éste tiene como objetivo realizar. En ese sentido, puede decirse que el grupo controla sus acciones y ordena las actividades de los miembros que lo componen de acuerdo a las opciones planteadas; así, si alguien falla en cumplir con su rol asignado, otro individuo puede reemplazarlo. Por otro lado, los individuos que conforman el grupo deben tener un control sensible de lo que están haciendo individualmente. Aún cuando no lo hagan en propio nombre, contribuyen a la acción grupal.

Para figurarlo, Pettit lo asimila al comportamiento de las moléculas de agua en punto de hervor. Una cantidad de agua $\mathrm{X}$ reventará el frasco que la contiene cuando se la expone a calor constante. Es irrelevante cuál es la molécula de agua que romperá en hervor primero e iniciaría dicho proceso, aunque es predecible que alguna lo hará. En el mismo sentido, pueden conocerse

la votación individual para garantizar consistencia) o resignar deliberación (no limitando cómo ni qué votan los agentes, y así no prevenir dicha inconsistencia).

La opción que cree válida -regular las decisiones individuales- lo lleva a sostener que deben dejarse algunas esferas del ente grupal bajo el control de los individuos a fin de evitar arbitrariedades. En primer lugar, para limitar las conductas inmorales o egoístas de la corporación. En segundo lugar, en temáticas vinculadas a la esfera privada de los miembros y tópicos especializados en los que se requiere una experticia cualificada para decidir (List \& Pettit, 2011, pp. 129-131). Para justificar este espacio de control individual frente a la corporación apela a una idea de libertad particular: libertad como no dominación o libertad republicana. En este punto, la tesis de Pettit es verdaderamente completa, pues se apoya sobre una idea de libertad política que justifica toda la práctica de responsabilidad de las comunidades y sus instituciones públicas. Ver: (Pettit, 1997a, 1997b, 1999, 2001b) 
Vercellone, A. L. ¿Puede la administración pública ser un agente autónomo? Una crítica a la teoría del órgano desde la filosofía moral. Derecho y Ciencias Sociales. Mayo-Octubre 2019 № 21. Pgs 166-185. ISNN 1852-2971. Instituto de Cultura Jurídica y Maestría en Sociología Jurídica. FCJ y S. UNLP

las actividades que el grupo tiene que realizar y controlarlas mediante mecanismos internos de organización. Alguno de sus miembros actuará para alcanzar esos objetivos, aunque es irrelevante quiénes lo haga: alguien lo hará, y si falla la estructura grupal permite reemplazar a dicho agente por otro que ordene mejor su conducta.

Este doble nivel de sensibilidad es lo que permite a Pettit atribuir responsabilidad a los entes, sin desconocer las influencias individuales en las acciones conjuntas.

\section{5. ¿Puede la administración pública ser un agente autónomo?}

Pettit desarrolla su tesis reconociendo que no toda actividad grupal es un agente moral autónomo. Sin dudas que un cuerpo legislativo con doble cámara y múltiples rondas de votación probablemente lo satisfaga. Pero esto no es extensible a cualquier área del gobierno, en especial cuando ésta se encuentra organizada burocráticamente. Ya que, es cuestionable que la administración pública sea un agente moral cuando en su estructura interna rigen principios como el de "división de tareas" y "organización por jerarquías". Actuando bajo esa estructura los burócratas suelen no ser conscientes del fin o la acción a la que contribuyen individualmente. E inclusive, suelen ignorar los resultados en los que redunda su acción desplegada en el pasado.

Para explicar mejor como estos dos principios representan un obstáculo para la responsabilidad colectiva, es útil concentrarse en el aspecto más crítico del argumento: la posibilidad de que el grupo tenga voluntad. Esto es, capacidad de razonamiento propio y superveniencia entre las voluntades individual y la grupal.

\section{a. Voluntad y jerarquías}

Al considerar la voluntad, Pettit sostiene que la posibilidad de tener creencias y deseos verdaderos sobre el mundo -diferentes e independientes de las de los individuos- depende de la estructura interna que adopta el grupo; en cuyo seno defiende un modo de organización democrática y deliberativa, y regulaciones específicas para la toma de decisiones.

Sin embargo, es dudoso que una institución pública burocrática pueda funcionar de ese modo, en especial cuando se caracteriza por tener una estructura interna jerárquica. En ese tipo de organizaciones los funcionarios de rango superior deciden las políticas a ejecutar y pueden controlar que éstas sean implementadas por los inferiores. Aún si se maximizan las cualidades de los agentes para las decisiones -es decir, aún si se garantiza que los agentes de la organización son infalibles y poseen creencias verdaderas sobre el mundo- la posibilidad de que la creencia del grupo sea verdadera no aumenta a medida que aumenta el número de miembros, pues ésta 
Vercellone, A. L. ¿Puede la administración pública ser un agente autónomo? Una crítica a la teoría del órgano desde la filosofía moral. Derecho y Ciencias Sociales. Mayo-Octubre 2019 № 21. Pgs 166-185. ISNN 1852-2971. Instituto de Cultura Jurídica y Maestría en Sociología Jurídica. FCJ y S. UNLP

depende en última instancia de un solo sujeto: su jerarca. Por lo tanto, las creencias del grupo serán o no verdaderas, según el criterio y falibilidad del funcionario superior.

El mismo Pettit sugiere esta conclusión cuando expresa que los grupos con toma de decisión dictatorial no pueden satisfacer todos los requisitos para la voluntad grupal (List \& Pettit, 2011, p. 53).

Y así, es difícil aceptar que pueda existir superveniencia entre voluntades. O mejor dicho, aunque es posible que las personas piensen con fines comunitarios y tengan en miras un fin común, las intenciones y deseos del grupo son las exclusivas de un único agente. Aún si se tratara de un sujeto infalible con la capacidad de pensar para la comunidad y no sólo en sus intereses particulares (por ejemplo, el jerarca mejor capacitado y públicamente orientado), la voluntad grupal está determinada por la voluntad de alguno de sus individuos o es dependiente de ella.

El problema está en que la intención primaria que reconocemos detrás de las acciones de la administración no pertenece a todos sus miembros. Puede darse que los individuos del grupo actúen sin pensar en fines particulares, compartan un objetivo en común y entiendan la dependencia contributiva de sus acciones -dados los roles que conocen y cumplen en la administración, es decir, es posible que satisfagan los requisitos para ejecutar una acción conjunta-. Pero la voluntariedad de dicha acción depende del tipo de racionalidad que pueden desplegar los miembros del grupo, como grupo. Esto es, el modo en que la decisión conjunta se nutre de las aportaciones individuales; y con ello, todo lo que el grupo conoce y desea acerca del mundo.

En otras palabras, el test de la superveniencia entre A y B que propone Pettit se supera cuando disponer los elementos de un hecho (B) modifica los de otro (A). En el caso de la burocracia jerárquica, modificar la conformación del grupo de burócratas de menor rango de la administración resulta irrelevante para la conformación de la voluntad grupal. Especialmente, si se considera que las decisiones de los funcionarios inferiores son impersonales y cualquier agente que circunstancialmente desempeñe el cargo debiera actuar en el mismo sentido obedeciendo las órdenes del superior-. Sólo la alteración del funcionario jerarca puede torcer la voluntad grupal. De hecho, parte importante de la idea de jerarquía burocrática implica el reconocimiento de poder discrecional de decidir sólo al jerarca, y al inferior el deber de acatar y ejecutar (Weber, 1977, 1993). 
Vercellone, A. L. ¿Puede la administración pública ser un agente autónomo? Una crítica a la teoría del órgano desde la filosofía moral. Derecho y Ciencias Sociales. Mayo-Octubre 2019 № 21. Pgs 166-185. ISNN 1852-2971. Instituto de Cultura Jurídica y Maestría en Sociología Jurídica. FCJ y S. UNLP

\section{b. $\quad$ Voluntad y división de tareas}

La división de tareas presenta un obstáculo similar, pues no contribuye para la alineación de una única voluntad para el grupo. O dicha formación no sucede cuando ésta no responde a un tipo de organización democrática que pueda garantizar que la relación entre las voluntades de los individuos -creencias, deseos e intenciones- supervenga en la del grupo.

Esto cobra sentido si se piensa en las actividades administrativas que generan responsabilidad. Lo que verdaderamente encontramos detrás de los errores o problemas de la administración son actividades negligentes, descuidos y omisiones de los funcionarios, y no la intención directa de causar un daño por parte de la administración. A menos que exista una particular connivencia en dichas actitudes, sería un absurdo decir que "la negligencia del conjunto de funcionarios superviene en una administración negligente". Funcionarios incompetentes posibilitan una administración que funciona con retardo, que es ineficaz y eventualmente causa daños. Pero no diremos que la administración -como ente- tiene la voluntad de actuar de ese modo. Sucede por el conjunto de faltas particulares, pero no por la convergencia de una voluntad común de dañar.

\section{c. $\quad$ El carácter antidemocrático de la burocracia}

En definitiva, el problema es que la burocracia no ha sido pensada para funcionar como un ente que decide democráticamente. $\mathrm{Y}$ el estructuralismo exige una organización de ese tipo para que el grupo pueda ser visto como algo más que la mera suma de voluntades individuales.

Las ventajas que se atribuyen a la burocracia -celeridad, eficiencia- dependen de que las decisiones se adopten de forma rápida por agentes cualificados y se ejecuten con eficacia por otros. Para ello son necesarias la existencia de jerarquías, facultades discrecionales escalonadas, división de tareas, y con ello, diferenciación entre deberes y responsabilidades en virtud del rango (Weber, 1977). En cuyo caso, no todos los agentes individuales pueden tener control sobre lo que hace o decide el ente. Así, la posibilidad de que exista responsabilidad moral grupal es implausible.

Esto no significa que debamos pensar a la burocracia como un órgano exclusivamente dictatorial; sobre todo si consideramos que sus reglamentos y límites son en general establecidos por un órgano democrático (como lo es el Congreso), donde las reglas de la democracia juegan un papel relevante; o que el ingreso a los cargos públicos respeta la igualdad de oportunidades. Pero estas regulaciones no significan que la institución constituya un agente moral. 
Vercellone, A. L. ¿Puede la administración pública ser un agente autónomo? Una crítica a la teoría del órgano desde la filosofía moral. Derecho y Ciencias Sociales. Mayo-Octubre 2019 № 21. Pgs 166-185. ISNN 1852-2971. Instituto de Cultura Jurídica y Maestría en Sociología Jurídica. FCJ y S. UNLP

\section{6. ¿Qué implicancia jurídica tienen estas consideraciones sobre el estructuralismo y la teoría del órgano? Algunas conclusiones}

A menos que una organización colectiva adopte una estructura interna democrática y posea regulaciones que impidan inconsistencias, entonces las acciones y decisiones del grupo son determinadas por uno o varios de sus miembros con prescindencia de los demás. Cuando se consideran seriamente los requisitos y dificultades existentes para configurar una verdadera estructura grupal, entonces es lógico dudar que una institución esencialmente verticalista -como la burocracia administrativa- pueda ser una persona con voluntad propia.

Las implicancias de esta reflexión para un sistema legal son varias. En primer lugar, si es implausible la agencia moral colectiva, entonces lo único que puede verse detrás de las actividades de la administración es un conjunto de acciones individuales. En el mejor de los casos, voluntades direccionadas en un mismo objetivo común (cuando las personas que se desempeñan en su interior tienen una verdadera vocación pública, y se preocupan por beneficiar a la colectividad). Pero siempre ordenadas por principios que indican a unos que deben seguir directivas, y a otros que tienen la potestad de decidir e impartir órdenes. Una sanción jurídica grupal probablemente ataca injustamente a aquellos que no tienen bajo su control la toma y ejecución de decisiones. Asimismo, puede desligar de responsabilidad a los jerarcas o funcionarios de la institución que son quienes verdaderamente controlan dichas actividades.

En segundo lugar, si el desempeño normal y regular de la administración no siempre responde al juicio de un agente racional, la tasa de daños que ocasione por su actividad lícita es previsible que sea mucho mayor del que las personas puedan anticipar. Cuando se reconocen las voluntades individuales que verdaderamente están detrás de los entes, puede apreciarse que muchas malas rutinas de la administración pública (demoras, retardo, ineficacia, etc.) son el resultado de malas decisiones o negligencias individuales, es decir, del mal desempeño de sus funcionarios públicos, pero nunca de un acuerdo colectivo de dañar. En definitiva, la responsabilidad colectiva deja también impunes a aquellos que son los verdaderos ideólogos de las faltas de la administración. Y la tercer y más importante implicancia es que es necesario reconsiderar a la teoría del órgano como teoría que justifica los supuestos de responsabilidad jurídica colectiva. Pues dicha teoría se apoya en un enunciado estructuralista fundamental -que la voluntad grupal es autónoma y diversa de la de sus miembros-, cuya plausibilidad requiere de una organización interna cuidadosamente diseñada. Si admitimos que la administración pública no se ha configurado de acuerdo a ella, entonces en su interior no hay más que la suma de voluntades individuales, la institución es un instrumento artificial, y la teoría del órgano no puede explicarla. 
Vercellone, A. L. ¿Puede la administración pública ser un agente autónomo? Una crítica a la teoría del órgano desde la filosofía moral. Derecho y Ciencias Sociales. Mayo-Octubre 2019 № 21. Pgs 166-185. ISNN 1852-2971. Instituto de Cultura Jurídica y Maestría en Sociología Jurídica. FCJ y S. UNLP

Aunque el legislador no la ha asumido expresamente, para la mayoría de los juristas el organicismo constituye uno de los principales argumentos para explicar y justificar la responsabilidad de las personas jurídicas. De manera que "la teoría del órgano no es más que el conjunto de opiniones de los juristas"(Echevesti, 2003). Dudar de dicha opinión, no implica negar la existencia de responsabilidad colectiva. Sino asumir que debe buscarse su raíz en alguna otra tesis que sea más adecuada para explicar los verdaderos vínculos que las personas entablan dentro de las organizaciones colectivas, y en especial, en una de particularidades tan llamativas como la administración pública.

En ese sentido, puede pensarse un buen número de razones para atribuir responsabilidad a los grupos. Por mencionar algunas: razones de utilidad (por ejemplo, que es útil para prevenir futuros daños causados por las organizaciones colectivas), de justicia (cuando se considera que las personas no pueden costear individualmente los daños causados por la administración, un régimen de exclusiva responsabilidad individual dejaría a muchos afectados sin resarcimiento por daños sufridos), o la propia finalidad del Estado (pues algunos entienden que dado el objetivo de realizar bienes públicos, los resultados de la actividad estatal debieran ser asumidos por la generalidad de sus beneficiarios). Todas ellas llevan a reconocer algún régimen de responsabilidad colectiva. Sin embargo, no implican la controvertida asunción de que la administración pública sea un órgano con autonomía propia.

Reconocer agencia moral a los grupos supondría el reconocimiento también de autonomía, con la misma extensión y similares efectos que a las personas humanas (Thompson, 1999, p. 115). La idea de "autonomía" implica tratar a la institución como un fin en sí mismo, es decir, como un agente que no puede ser utilizado como un mero medio o un objeto para alcanzar fines de otros. En la filosofía tradicional, esto supone que los agentes merecen un trato digno, que les permite desarrollarse como personas (Kant, 2000, p. 88). Esto último resulta ilógico si pensamos que la institución no es más que una herramienta creada para un fin particular. Como señala Thompson, la consecuencia más grave de conceder este tipo de autonomía a los entes colectivos es que no podría realizarse un apropiado control sobre las instituciones públicas. Apelando a su autonomía, del mismo modo que pueden hacerlo las personas físicas, las instituciones podrían reclamar una esfera de no regulación o no interferencia por parte de terceros (Thompson, 1999, p. 115).

Estas reflexiones no niegan que la práctica de la responsabilidad sea posible en las instituciones colectivas. Sino meramente que, la administración pública no puede ser un agente moral autónomo. Aunque puede ser deseable un régimen colectivo de responsabilidad, es necesario llamar la atención sobre los múltiples presupuestos y verdaderas consecuencias que esto 
Vercellone, A. L. ¿Puede la administración pública ser un agente autónomo? Una crítica a la teoría del órgano desde la filosofía moral. Derecho y Ciencias Sociales. Mayo-Octubre 2019 № 21. Pgs 166-185. ISNN 1852-2971. Instituto de Cultura Jurídica y Maestría en Sociología Jurídica. FCJ y S. UNLP

significa. En ese sentido, este artículo no pretende negar la responsabilidad colectiva legal, ni la utilidad de las instituciones colectivas, sino argumentar en contra de una teoría que goza de amplio acuerdo en el medio jurídico, pero que es inaplicable para el régimen público: la teoría del órgano. Y finalmente, quizás pensar que debe abogarse por una teoría explicativa más apropiada para las instituciones públicas, y un sistema de responsabilidad que también busque dentro de la administración pública por los agentes individuales que causan daños o cometen delitos.

\section{Bibliografía.}

Andrada, A. D. (2010). Nuevas problemáticas sobre la responsabilidad del Estado. LA LEY 24/08/2010, 1 .

Cassagne, J. C. (2005). Reflexiones sobre los factores de atribución en la responsabilidad del Estado por la actividad de la administración. $L A L E Y$.

Cesano, J. D., \& Balcarce, F. I. ( 2003). Reflexiones sobre la responsabilidad penal de las personas jurídicas en la República Argentina.POENALIS No. 5 Marzo.

Echevesti, C. (2003). Responsabilidad de los funcionarios públicos. Bs As: Hammurabi.

Espí, C. J. M. (2011). Filosofía de la mente. Universitat de València.

Feinberg, J. (1970). Doing \& deserving: Princeton UP.

Hevia, M. (2014). Tres visiones de la responsabilidad del Estado y de sus funcionarios públicos en el nuevo Cógido Civil y Comercial. Tiempo Judicial. Recuperadohttp://www.tiempojudicial.com/web/TiempoJudicial/diarios-pdf

Ivanega, M. M. (2005). Las responsabilidades de los funcionarios públicos. Biblioteca Jca. Virtual del Instituto de Investigaciones Jurídicas de la UNAM. México DF.

Kant, I. (2000). Crítica de la razón pura. Valencia: Universitat de Valencia.

List, C., \& Pettit, P. (2005). Group agency and supervenience. The Southern journal of philosophy, 44(Supplement), 85-105.

List, C., \& Pettit, P. (2011). Group agency: The possibility, design, and status of corporate agents: Oxford University Press Oxford.

Palazzo, J. L. (1995). Responsabilidad extracontractual del Estado. Bs As: Depalma.

Pettit, P. (1996). The common mind: An essay on psychology, society, and politics: Oxford University Press.

Pettit, P. (1997a). Republicanism: a theory of freedom and government: OUP Oxford. 
Vercellone, A. L. ¿Puede la administración pública ser un agente autónomo? Una crítica a la teoría del órgano desde la filosofía moral. Derecho y Ciencias Sociales. Mayo-Octubre 2019 № 21. Pgs 166-185. ISNN 1852-2971. Instituto de Cultura Jurídica y Maestría en Sociología Jurídica. FCJ y S. UNLP

Pettit, P. (1997b). -Republicanismo, Una Teoría sobre el Gobierno y la Libertad. Barcelona: Paidós.

Pettit, P. (1999). Republican freedom and contestatory democratization. Democracy's value, 163-190.

Pettit, P. (2001a). Deliberative democracy and the discursive dilemma. Philosophical Issues, 11(1), 268-299.

Pettit, P. (2001b). A theory of freedom: from the psychology to the politics of agency: Oxford University Press on Demand.

Pettit, P. (2003). Deliberative democracy, the discursive dilemma and republicanism. Philosophy politics and society.

Pettit, P. (2007). Responsibility Incorporated*. Ethics, 117(2), 171-201.

Pettit, P., \& Schweikard, D. (2006). Joint actions and group agents. Philosophy of the Social Sciences, 36(1), 18-39.

Pizarro, D. (2016). Responsabilidad del Estado y del funcionario público (Vol. I): Astrea.

Risser, D. T. (2006). Collective Moral Responsibility. Internet Encyclopedia of Philosophy (Accessed 8 Sept 2007).

Robiglio, C. (2007). Responsabilidad penal de las personas jurídicas por contrabando. Comentario del fallo Fly Machine SRL. Revista del Centro Argentino de Estudios en lo Penal Tributario.

Thompson, D. F. (1999). La ética política y el ejercicio de cargos públicos: Gedisa.

Thompson, D. F. (2004). Restoring responsibility: ethics in government, business, and healthcare (Vol. 575): Cambridge University Press.

Trigo Represas, F. A. (2009). Tratado jurisprudencial y doctrinario (Vol. 1). Bs. As: LA LEY. Valdivia Olivares, J. M. (2006). Teoría del órgano y responsabilidad pública en la Ley de Bases de la Administración del Estado. Revista de derecho, 19(2), 133-159.

Weber, M. (1977). Qué es la burocracia? : La Pléyade.

Weber, M. (1993). Economía y sociedad. Mexico: Fondo de cultura económica. 